\title{
The length-difficulty law of serial learning: An empirical function and a theoretical derivation
}

WILLIAM BOGARTZ AND ARLENE HEADRICK

UNIVERSITY OF KANSAS

\begin{abstract}
List length, item meaningfulness, and presentation rate were varied in a factorially designed serial learning experiment. In all conditions, the function relating length ( $r$ ) with trials to criterion ( $t$ ) was of the form $\mathrm{t}=\mathrm{a}+\mathrm{b} \log \mathrm{r}$. Mathematical analysis revealed that such a function is implied by the classic negatively accelerated exponential mean learning curve. A theoretical expression for total errors to criterion as a linear function of list length was also derived and confirmed.

\section{Problem}

The effect of length of a serial list on the number of repetitions necessary for acquisition has been subject to more speculation than study. The relationship has been said to be linear (Waugh, 1962), a square root function (Thurstone, 1930), disproportionate (Osgood, 1953), roughly proportional (Hovland \& Kurtz, 1952), and not necessarily disproportional (Deese, 1958). The purpose of this study was to investigate the length-difficulty function under a controlled range of conditions, in order to lay the groundwork for mathematical treatment. Method
\end{abstract}

A serial list of $5,10,15$, or $30 \mathrm{CVCs}$ of high (mean $\mathrm{m}^{\prime}=4.06$ ) or low (mean $\mathrm{m}^{\prime}=2.25$ ) meaningfulness (Noble, 1961) was learned by each of 64 undergraduate Ss at either a $2 \mathrm{sec}$. or a $4 \mathrm{sec}$. rate. Stimuli were presented in a Lafayette model 303A memory drum to a criterion of one perfect trial by the method of paced anticipation. Each $\mathrm{S}$ learned one list. Four Ss were run in each of the 16 combinations of length, $\mathrm{m}^{\prime}$, and rate. Syllables for the shorter lists at each level of meaningfulness were selected at random from the 30 items comprising the longest list.

\section{Results}

Figure 1 shows mean trials to criterion as a function of list length for each combination of $\mathrm{m}^{\prime}$ and rate. Fitting a common regression function of the form $t=a+b \log r$, where $r$ is the number of items, accounted for .998 of the variation attributable to list length, $F(1,48)=99.09, p<.0001$. The component for $\log r$ interacted significantly with rate, $F(1,48)=11.22, p<.005$; and with the interaction of meaningfulness and rate, $F(1,48)=4.06, p<.05$; but not quite significantly with meaningfulness, $F(1,48)=2.89, .05<p<.10$. Rate and meaningfulness thus affected the slope of the regression function, but the effect of the latter variable was manifested only at the faster rate of presentation.

In no case were there significant deviations from the common regression function or from any of the separate regression functions shown as smooth curves in Fig. 1
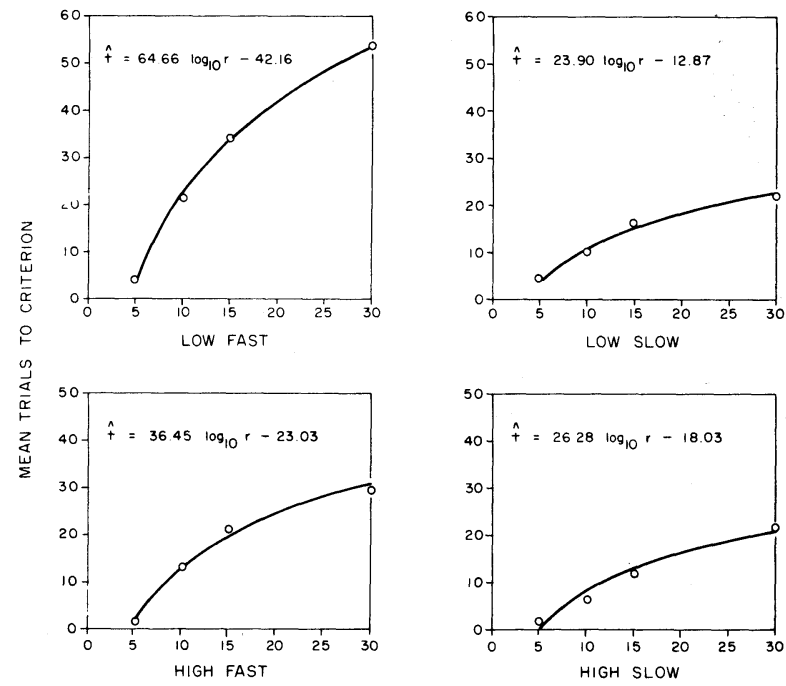

Fig, 1. Mean trials to criterion as a function of list length.

(highest $\mathrm{F}=. \mathrm{.13}$ )。It is therefore clear that the form of the length-difficulty function remained invariant over the conditions studied.

The complete analysis of variance also yielded significant effects of meaningfulness, $F(1,48)=10.86$, $\mathrm{p}<.005$; rate, $\mathrm{F}(1,48)=24.29, \mathrm{p}<.001$; meaningfulness by rate, $F(1,48)=4.41, p<.05$; length, $F(3,48)=33.10$, $\mathrm{p}<.001$; and rate by length, $\mathrm{F}(3,48)=3.79, \mathrm{p}<.025$. Trials decreased with item meaningfulness and increased with faster presentation rate and list length, but the rate effect was greater for low meaningfulness items or for longer lists, and the meaningfulness effect was appreciable only at the faster rate.

\section{Diseussion}

The above results strongly suggest that the function $t=a+b \log r$ may be regarded as the length-difficulty law of serial learning. As a partial test of generality, the function was also fitted to data from Cofer (1941) for verbatim learning of English text, and to two sets of data from Ebbinghaus (1885, pp. 46-49) for nonsense syllables learned by the method of whole presentation. In each case, as shown in Fig. 2, the regression accounted for more than .99 of the variation attributable to list length.

It is possible that the proposed length-difficulty law may have considerable generality. The analysis which follows will show that any process which produces a classic exponential learning curve also implies a length difficulty function similar to the one obtained above.

Such a process may be described most simply by the (deterministic) linear difference equation, 

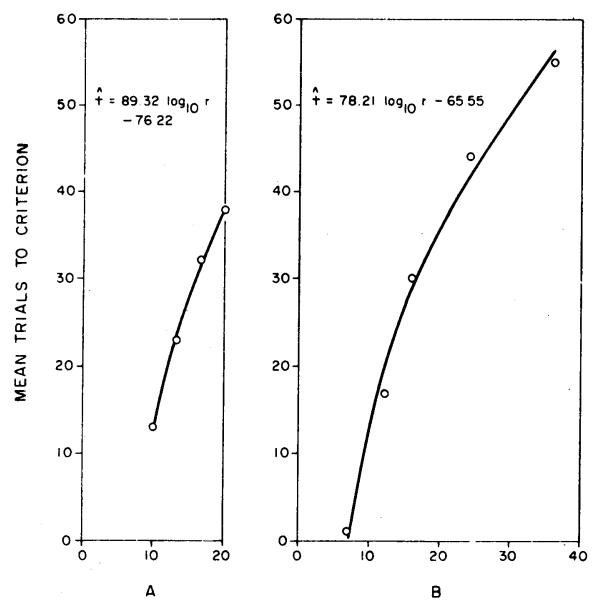

(1) $e_{n}+1=(1-\theta) e_{n}$

which has the solution,

$$
\text { (2) } e_{n}=e_{1}(1-\theta)^{n-1}
$$

where $e_{n}$ is the mean, or "true" number of errors on the $\mathrm{n}^{\text {th }}$ trial. This is equivalent to the mean learning curve which arises in a number of stochastic models. Taking logarithms and solving for $(n-1)$, we find

$$
\text { (3) } n-1=b\left(\log e_{1}-\log e_{n}\right)
$$

where

$$
b=-1 / \log (1-\theta)
$$

If it is now assumed that (a) on the first trial an error is made on every item $\left(e_{1}=r\right)$, and $(b) t$ is the mean number of trials to a fixed "criterion" $\left(e_{t+1}=\right.$ constant), then (3) becomes

$$
\text { (4) } t=b \log r-a, \text { Q.E.D. . }
$$
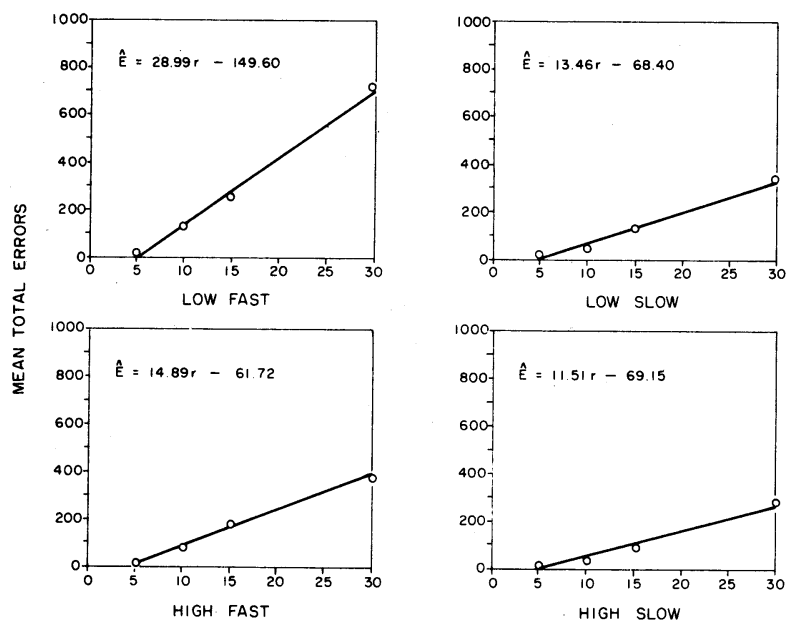

LOW SLOW

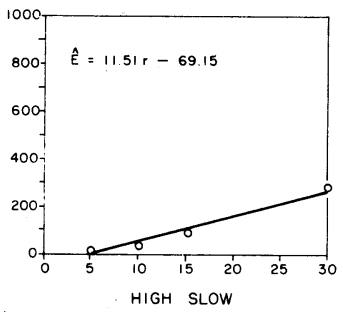

Fig. 3. Mean total errors as a function of list length.
Fig. 2. Mean trials to criterion as a function of amount of material: (A) Data from Ebbinghaus (1885), earlier period; (B) Data from Ebbinghaus (1885), later period; (C) Data from Cofer (1941).
Also, summing (2) over the first t trials, we obtain for mean total errors

$$
\text { (5) } E=\left(r-e_{\dagger}\right) / \theta
$$

Equation (5) states that total errors are linearly related to list length, a proposition which was unexpected prior to the analysis, and therefore provides a further test of the theory. Least-squares straight lines fitted to mean total errors as a function of list length are presented in Fig. 3.

It is clear that the deterministic model described above provides a good first approximation to the lengthdifficulty problem. Since the exponential learning curve is consistent with a broad class of stochastic processes and is known to fit many experimental situations reasonably well, it is likely that the length-difficulty law will be found to be quite general, within the limitations of the assumptions made.

Of the assertions of previous authors concerning the length-difficulty relationship, only the function proposed by Thurstone (1930) remains as a serious alternative. However, because the Thurstone function derives from an inappropriate sigmoid learning curve and requires estimation of two parameters to achieve a comparable fit to the data, and also for reasons of simplicity and relevance to current theory, the present law appears to be preferable.

\section{References}

Cofer, C. N. A comparison of logical and verbatim learning of prose passages of different lengths. Amer. J. Psychol., 1941, 54, 1-16.

Deese, J. The psychology of learning. New York: McGraw-Hill, 1958.

Ebbinghaus, H. Memory. Ner: York: Teachers' College, 1913. Hovland, C.. I., \& Kurtz, K. H. Experimental studies in rote-learning theory: X. Pre-learning syllable familiarization and the lengthdifficulty relationship. J. exp. Psychol., 1952, 44, 31-39.

Osgood, C. E. Method and theory in experimental psychology. New York: Oxford, 1953.

Thurstone, L. L. The learning function. J. gen. Psychol., 1930, 3, 469-493.

Waugh, Nancy C. Length of series and the learning curve. Amer. $J$. Psychol., 1962, 75, 177-192. 